Mens

Revue d'histoire intellectuelle et culturelle

smens

\title{
La commission BB et la bureaucratie fédérale
}

\section{Daniel Bourgeois}

Volume 14-15, numéro 2-1, printemps-automne 2014

La Commission royale d'enquête sur le bilinguisme et le biculturalisme a 50 ans : période révolue ou projet inachevé ?

URI : https://id.erudit.org/iderudit/1035526ar

DOI : https://doi.org/10.7202/1035526ar

Aller au sommaire du numéro

\section{Éditeur(s)}

Centre de recherche en civilisation canadienne-française

\section{ISSN}

1492-8647 (imprimé)

1927-9299 (numérique)

Découvrir la revue

Citer cet article

Bourgeois, D. (2014). La commission BB et la bureaucratie fédérale. Mens, 14-15(2-1), 13-51. https://doi.org/10.7202/1035526ar

\section{Résumé de l'article}

La commission BB fut établie pour étudier le bilinguisme et le biculturalisme, plus particulièrement dans l'administration fédérale, et recommander des mesures pour l'améliorer et être en mesure de maintenir l'unité nationale. Les deux tiers de ses 150 recommandations portaient sur l'appareil fédéral, et plusieurs proposaient un chambardement majeur. Selon la Commission, les autres mesures administratives adoptées au compte-gouttes ne suffisaient plus et des mesures symboliques, notamment la création de districts bilingues et d'unités de langue française, étaient requises pour que l'appareil fédéral reflète et nourrisse le bilinguisme et le biculturalisme partout au Canada. On y décèle une certaine méfiance envers la bureaucratie fédérale " anglophone " ainsi qu'un manque de confiance dans sa capacité d'innover afin de relever les défis existentiels du Canada. Sa méfiance et son manque de confiance furent à la fois confirmés et infirmés par la suite. D’une part, en même temps que la Commission effectuait son travail, l'appareil fédéral proposait des mesures qui rejoignaient celles que la Commission présentera plus tard et qui les ont quasiment rendues désuètes. D'autre part, l'appareil a discrètement saboté la " pierre angulaire » de ses recommandations - les districts bilingues - parce que, n’appréciant pas leur valeur symbolique, il a préféré mettre en place, entre-temps, ses propres mesures administratives. De plus, certaines autres recommandations se sont révélées problématiques. Nous en tirons deux leçons. D'abord, la Commission avait une bonne compréhension de l'appareil fédéral. Ensuite, la structuration de la mise en oeuvre des mesures administratives a contribué à l'échec des districts bilingues.
Ce document est protégé par la loi sur le droit d'auteur. L'utilisation des services d'Érudit (y compris la reproduction) est assujettie à sa politique d'utilisation que vous pouvez consulter en ligne.

https://apropos.erudit.org/fr/usagers/politique-dutilisation/ 


\title{
La commission $B B$ et la bureaucratie fédérale
}

\author{
Daniel Bourgeois \\ Institut Beaubassin
}

\section{Résumé}

La commission BB fut établie pour étudier le bilinguisme et le biculturalisme, plus particulièrement dans l'administration fédérale, et recommander des mesures pour l'améliorer et être en mesure de maintenir l'unité nationale. Les deux tiers de ses 150 recommandations portaient sur l'appareil fédéral, et plusieurs proposaient un chambardement majeur. Selon la Commission, les autres mesures administratives adoptées au compte-gouttes ne suffisaient plus et des mesures symboliques, notamment la création de districts bilingues et d'unités de langue française, étaient requises pour que l'appareil fédéral reflète et nourrisse le bilinguisme et le biculturalisme partout au Canada. On y décèle une certaine méfiance envers la bureaucratie fédérale " anglophone " ainsi qu'un manque de confiance dans sa capacité d'innover afin de relever les défis existentiels du Canada. Sa méfiance et son manque de confiance furent à la fois confirmés et infirmés par la suite. D’une part, en même temps que la Commission effectuait son travail, l'appareil fédéral proposait des mesures qui rejoignaient celles que la Commission présentera plus tard et qui les ont quasiment rendues désuètes. D'autre part, l'appareil a discrètement saboté la " pierre angulaire " de ses recommandations - les districts bilingues - parce que, n'appréciant pas leur valeur symbolique, il a préféré mettre en place, entre-temps, ses propres mesures administratives. De plus, 
certaines autres recommandations se sont révélées problématiques. Nous en tirons deux leçons. D'abord, la Commission avait une bonne compréhension de l'appareil fédéral. Ensuite, la structuration de la mise en œuvre des mesures administratives a contribué à l'échec des districts bilingues.

\section{Abstract}

The B\&B Commission was established to study bilingualism in the federal public service and to recommend measures to improve bilingual services in order to ensure and promote national unity. Two thirds of its 150 recommendations dealt with the federal apparatus and many proposed sweeping changes. For the Commission, minor changes were no longer satisfactory and significant measures, including the creation of bilingual districts and French-language work units, were required so that the public service could reflect and nourish bilingualism and biculturalism throughout Canada. Many recommendations expressed a fundamental mistrust of the "Anglophone" bureaucracy and a lack of faith in its capacity to meet Canada's existential challenges. The Commission's mistrust would be at once confirmed and dispelled by the subsequent actions of the public service. On the one hand, while the commissioners' work was ongoing, the federal apparatus itself put forward a number of changes which the Commission would later recommend. On the other hand, the public service discretely sabotaged the "cornerstone" of the Commission's recommendation, bilingual districts, because it did not appreciate their symbolic value and preferred the incremental measures that had already implemented. Moreover, other recommendations proved problematic. Two lessons can thus be drawn. Firstly, that the B\&B Commission had a good understanding of the federal apparatus and, secondly, that the structured implementation of administrative change facilitated the failure of bilingual districts. 
La Commission royale d'enquête sur le bilinguisme et le biculturalisme fut établie en 1963 en grande partie pour " faire rapport sur l'état et la pratique du bilinguisme dans tous les services et institutions de l'administration fédérale [...] et présenter des recommandations de nature à assurer le caractère bilingue et fondamentalement biculturel de l'administration fédérale ${ }^{1}$ ». Ses membres ont proposé 150 mesures à cet effet, dont 109 portaient sur l'appareil fédéral. Les 41 autres recommandations s'adressaient aux provinces et aux entreprises privées.

On peut diviser ces 109 recommandations en cinq catégories. Sept d'entre elles portaient sur les districts bilingues. Vingt-neuf proposaient de modifier les structures administratives fédérales. Dixhuit recommandations concernaient l'éducation en milieu minoritaire. Dix-sept autres proposaient le soutien fédéral aux provinces. Enfin, 38 portaient sur des mesures administratives internes. Six des 109 recommandations chevauchaient deux catégories.

Notre texte décrit les 109 recommandations d'ordre administratif et la connaissance qu’avait la Commission de l'appareil fédéral. Il explique pourquoi certaines recommandations se sont révélées problématiques. Nous en tirons quelques leçons concernant la structuration administrative.

\section{Lappareil fédéral selon la Commission}

La genèse de la Commission royale d'enquête sur le bilinguisme et le biculturalisme remonte aux refus, hésitations et tergiversations de l'appareil fédéral en la matière. Dans ses chroniques et éditoriaux, André Laurendeau se plaint du caractère discret, marginal et

\footnotetext{
${ }^{1}$ Commission royale d'enquête sur le bilinguisme et le biculturalisme (ci-après Commission BB), Rapport de la Commission royale d'enquête sur le bilinguisme et le biculturalisme (ci-après Rapport), vol. 1 : Les langues officielles, Ottawa, Imprimeur de la reine, 1967, p. 180. Le rapport de la Commission est disponible en version numérique sur le site de Bibliothèque et Archives Canada : [http://epe.lac-bac. gc.ca/100/200/301/pco-bcp/commissions-ef/dunton1967-1970-ef/dunton196770-fra.htm].
} 
" artisanal " des mesures fédérales, ce qui l'incite à revendiquer "un système d'ensemble $e^{2}$ » et " une réforme autrement générale ${ }^{3}$ ». Selon lui, seule " une enquête royale sur le fonctionnement de l'usine " peut déterminer ce qu'est le bilinguisme canadien, "ce qu'il devrait être, ce qu'il pourrait devenir ${ }^{4}$ ».

Une fois en place, la Commission a vite compris le rôle déterminant de l'appareil fédéral dans la prise de décision et a commandé plusieurs recherches sur son fonctionnement ${ }^{5}$, qu'elle a ensuite abordées lors de plusieurs réunions. Son analyse de l'appareil fédéral est cinglante : «Il n’y a pas de véritable égalité entre francophones et anglophones dans la fonction publique fédérale ${ }^{6}$. " La discrimination contre le français ne peut plus continuer sans saper davantage "l'harmonie entre les deux groupes et l'existence même du Canada ${ }^{7}$ ". En effet, « l'intérêt de l'unité nationale » exige l'usage des deux langues dans l'administration fédérale ${ }^{8}$. Et pourtant, malgré plusieurs efforts, les résultats ont un "caractère fortuit et limité " ". Il fallait donc adopter une politique d'ensemble appliquant le " principe d'égalité " des deux peuples au sein de la fonction publique fédérale ${ }^{10}$.

2 André Laurendeau, "Devant des refus ", Le Devoir, 8 février 1962, p. 4.

3 André Laurendeau, "Pour une enquête sur le bilinguisme ", Le Devoir, 20 janvier 1962 , p. 4.

4 Laurendeau, "Devant des refus », p. 4.

5 C. E. S. Franks, Bilingualism and Biculturalism in the Federal Treasury Board: a Staff Report for the Royal Commission on Bilingualism and Biculturalism, Ottawa, Royal Commission on Bilingualism and Biculturalism, 1966; John Heward, History of Bilingualism and Biculturalism in the Canadian Public Service, Ottawa, Royal Commission on Bilingualism and Biculturalism, 1966; Jacques-Yvan Morin, Le fédéralisme canadien et le principe de l'égalité des deux nations, Ottawa, Commission royale d'enquête sur le bilinguisme et le biculturalisme, 1966; Claude-Armand Sheppard, The Law of Languages in Canada, Ottawa, Royal Commission on Bilingualism and Biculturalism, 1970.

${ }^{6}$ Commission BB, Rapport, vol. 3 : Le monde du travail, p. 275.

7 Ibid., p. 115.

8 Ibid., p. 119.

9 Ibid., p. 186.

${ }^{10}$ Ibid., p. 187. 
La Commission s'est inspirée des propos d'Eugène Therrien, un des trois membres de la Commission royale d'enquête sur l'organisation du gouvernement (commission Glassco). Offusqué par le refus de ses deux collègues anglophones d'aborder plus à fond la question linguistique au sein de l'appareil fédéral, il a rédigé un commentaire de onze pages intitulé « La question du bilinguisme dans l'administration fédérale ". Selon lui, le bilinguisme " n'est pas traité dans l'administration fédérale canadienne comme il devrait l'être, c'est-àdire comme un instrument d'administration efficace ${ }^{11}$ ». Au lieu de cela, on le réduit à la traduction, de l'anglais vers le français ${ }^{12}$. Therrien se plaint aussi, entre autres, de l'absence de statistiques sur le nombre de francophones dans la fonction publique fédérale, de l'absence du français dans les communications internes, même au Québec, du faible nombre de francophones détenant des postes de direction, de l'assimilation des francophones au sein des Forces armées et du délai trop long pour rendre disponible la version française des documents gouvernementaux ${ }^{13}$. En comparant le bilinguisme administratif avec celui d'autres pays, il constate que les efforts du Canada ne sont ni adéquats ni satisfaisants ${ }^{14}$. Entre autres, il suggère de recruter davantage de fonctionnaires francophones, surtout aux échelons supérieurs, d'exiger que le bilinguisme soit une " qualification nécessaire pour tous les employés en contact avec le public dans les villes et les régions bilingues ", de faire du français une langue de travail dans l'administration fédérale, y compris dans les Forces armées, d'améliorer la qualité et la quantité de l'information destinée au public en français

11 Eugène Therrien, "La question du bilinguisme dans l'administration fédérale ", dans Commission royale d'enquête sur l'organisation du gouvernement, Rapport, t. 1 : La gestion de la fonction publique, Ottawa, Imprimeur de la reine pour la Commission royale d'enquête sur l'organisation du gouvernement, 1962, p. 77. Le rapport de la Commission est disponible en version numérique sur le site de Bibliothèque et Archives Canada : [http://epe.lac-bac.gc.ca/100/200/301/pcobcp/commissions-ef/glassco1962-fra/glassco1962-fra.htm].

12 Ibid., p. 72.

13 Ibid., p. 73-74.

14 Ibid., p. 75. 
et de confier la question du bilinguisme au Conseil du trésor, qui amorçait son ascension dans le processus décisionnel du gouvernement fédéral ${ }^{15}$. Sa conclusion est sans équivoque ${ }^{16}$ : les francophones $\mathrm{du}$ pays ne doivent plus être considérés comme des « citoyens de seconde zone " par la fonction publique nationale.

Ses propos inspirent aussi le gouvernement Pearson. Avant même de créer la commission BB, il crée un comité spécial de hauts fonctionnaires pour en assurer le suivi. Ce comité se penchera sur les cours de langue, le recrutement, la formation, l'avancement et l'affectation du personnel, la création d'un institut fédéral du bilinguisme et d'un service du bilinguisme dans les ministères, la prime au bilinguisme, l'utilisation de formulaires, manuels et circulaires bilingues et le français au sein des Forces armées. En découlera une politique de bilinguisme, adoptée par la Commission du service civil le $1^{\text {er février }}$ 1966, qui se rapproche des recommandations de la commission BB, dont les premières seront publiées seulement vingt mois plus tard.

La politique veut favoriser et renforcer «l'unité nationale sur la base de l'égalité des droits et des chances pour les Canadiens, qu'ils soient d'expression anglaise ou d'expression française ${ }^{17}$ ». Elle prévoit l'utilisation des deux langues dans les communications internes, un recrutement accru de francophones, la création d'un climat propice au français au sein de l'appareil fédéral et des communications avec le public dans la langue officielle du client. Elle « doit s'appliquer graduellement sur une période de plusieurs années et d'une façon qui ne cause aucune injustice ni malentendu » et " en aucune façon porter préjudice à la carrière des fonctionnaires ${ }^{18}$ ". Quoi qu'il en

15 Ibid., p. 77-81.

16 Ibid., p. 74.

17 Lester B. Pearson, "Déclaration de principe sur le bilinguisme », 6 avril 1966, dans Canada, Compte rendu officiel des débats de la Chambre des communes, $27^{e}$ Législature, $1^{\text {re }}$ session, vol. 4 : $1966:$ du 29 mars au 27 avril 1966, Ottawa, Roger Duhamel, Imprimeur de la reine, 1966, p. 3915-3917, [En ligne], [http:// parl.canadiana.ca/view/oop.debates_CDC2701_04/1? r=0\&ss=2], image 589.

18 Ibid., p. 3916, image 590. 
soit, le bilinguisme devient l'un des "éléments d'appréciation dans le choix des candidats qui aspirent aux postes de la fonction publique ». La politique comprend également sept mesures spécifiques. Par exemple, le gouvernement établira " un programme spécial destiné à accroître le bilinguisme chez les fonctionnaires de haute direction qui exercent leurs fonctions dans la Capitale nationale ${ }^{19}$ ", offrira une prime au bilinguisme et élargira le programme de formation linguistique. Le gouvernement créera également un secrétariat spécial du bilinguisme en collaboration avec la Commission du service civil, le Conseil du trésor et les dirigeants d'organismes fédéraux pour sa mise en œuvre " coordonnée et progressive ». Enfin, elle donne un avantgoût des districts bilingues en faisant de la connaissance des deux langues un critère de qualification dans les 82 régions du pays où habite une minorité de langue officielle formant au moins $10 \%$ des habitants et comptant au moins 10000 personnes.

La politique sera appuyée en 1967 par la Loi concernant l'emploi dans la Fonction publique du Canada ${ }^{20}$. La loi comprend deux articles portant sur les langues officielles. L'article 12 affirme que la connaissance des deux langues est un critère de mérite au même titre que l'instruction et l'expérience, et l'article 20 stipule que la connaissance des deux langues est une condition d'embauche pour certains postes. Les règlements établissent des " régions bilingues " et les critères démolinguistiques et géographiques afférents ${ }^{21}$. Ces régions seront établies en fonction du même critère démolinguistique que les districts bilingues bientôt recommandés - $10 \%$ des habitants -, mais en fonction de l'aire de service des bureaux fédéraux plutôt que des unités de recensement, comme le proposera la Commission. Ainsi, les employés qui travaillent dans un bureau dont l'aire de service

19 Ibid.

${ }^{20}$ Loi concernant l'emploi dans la Fonction publique du Canada, 15 Elizabeth II (1967), chap. 71.

${ }^{21}$ Public Service Commission of Canada, Public Service Employment Act, dans The Canada Gazette, part II, vol. 101, n 7 : Statutory Orders and Regulations 1967, 12 avril 1967, Ottawa, Roger Duhamel, Imprimeur de la reine. 
comprend une minorité formant entre $40 \%$ et $60 \%$ de la population doivent être bilingues, alors que le nombre d'employés qui travaillent dans un bureau dont l'aire de service comprend une minorité qui représente entre $10 \%$ et $40 \%$ des habitants doit être proportionnel au pourcentage de la population minoritaire. L'article est muet sur les aires de service comprenant une minorité formant plus de $60 \%$ des habitants.

\section{Les recommandations de nature administrative}

La Commission a examiné les commentaires de Therrien et le suivi qu'en a fait le gouvernement Pearson, mais elle a finalement décidé de maintenir ses recommandations malgré le risque de les publier trop tard. Ses membres préconisaient des recommandations fondées sur leurs recherches et leurs délibérations plutôt que sur les décisions gouvernementales. Par ailleurs, ils ont gardé celles qui portaient sur les districts bilingues tripartites parce que les mesures prises par le gouvernement en 1966 et 1967 n'allaient pas assez loin et négligeaient leur grande valeur symbolique.

\section{Les districts bilingues}

Le district bilingue devait devenir la " pierre angulaire " du nouveau système $^{22}$. Cette unité administrative régionale établie là où la minorité linguistique représentait $10 \%$ de la population devait chambarder les appareils fédéral, provincial et municipal en raison de sa valeur symbolique ${ }^{23}$. D'abord, la désignation «bilingue" reconnaîtrait la présence des communautés francophones hors du Québec. Après un siècle de négligence, cette désignation leur donnerait un important appui moral. Ensuite, les districts bilingues devaient limiter l'usage de l'anglais au Québec aux régions où les anglophones étaient assez nombreux. Enfin, ils devaient encourager

22 Commission BB, Rapport, vol. 1 : Les langues officielles, p. 121.

23 Daniel Bourgeois, Canadian Bilingual Districts: From Cornerstone to Tombstone, Montréal, McGill-Queen's University Press, 2006. 
les gouvernements provinciaux et les municipalités à en faire un projet conjoint.

Le district bilingue avait également une valeur administrative : ses frontières, tracées à partir des données du recensement, devaient déterminer les régions du Canada où les communications et les services au public devaient être fournis dans les deux langues officielles. Les districts bilingues statutaires remplaceraient ainsi les régions bilingues établies en 1938 par règlements fédéraux ${ }^{24}$. Ces régions bilingues se limitaient aux endroits où la minorité linguistique formait la majorité de la population et avaient été réduites au minimum par une bureaucratie fédérale réticente. Elles étaient peu connues et négligeaient les fonctions provinciales et municipales. Le district bilingue aurait exigé que la minorité linguistique représente $10 \%$ de la population, aurait desservi la grande majorité des populations minoritaires de langue officielle, aurait encouragé la participation des institutions provinciales et municipales et aurait assuré que leur présence et leurs frontières soient bien connues.

Le district bilingue se présentait donc à la fois comme une amélioration du système administratif minimaliste en place et, surtout, comme un outil de reconnaissance du fait français hors Québec et de réduction de l'usage de l'anglais dans cette province. C'est sa valeur symbolique qui lui mérite le titre de "pierre angulaire ». Fondé sur la réalité géographique des minorités linguistiques plutôt que d'être laissé à la discrétion des bureaucrates, il transforme l'appareil administratif pour enrayer la crise de l'unité nationale. Cette recommandation fondamentale prenait appui sur plusieurs mémoires et recherches favorables à la mise sur pied de districts bilingues au Canada, inspirés du modèle finlandais ${ }^{25}$.

La commission $\mathrm{BB}$ adopte comme principe la "reconnaissance par la loi et dans la pratique des deux langues officielles, même là où

24 Canada, Loi modifiant la Loi du service civil, 2 George VI (1938), chap. 7, art. 1.

${ }^{25}$ Kenneth D. McRae, "Bilingual Language Districts in Finland and Canada: Adventures in the Transplanting of an Institution ", Analyse de politiques, vol. 4, no 3 (été 1978), p. 331-351, [En ligne], [http://www.jstor.org/stable/3549443]. 
l'une des deux est parlée par une minorité, dès que, numériquement, celle-ci paraît viable $e^{26} \%$. De ce fait, le critère déterminant pour la prestation des services bilingues est le même que celui qui détermine la viabilité d'une minorité : le nombre. Il fallait retenir des critères " objectifs", notamment démographiques et géographiques, qui forment ensemble "la carte démographique du Canada ", afin de ne « dispenser des services dans la langue de la minorité que dans la mesure où celle-ci les réclamerait ${ }^{27}{ }$. D'ailleurs, on ne pouvait fonder la prestation des services bilingues sur le principe de la « demande suffisante " parce qu'un tel système "ne comporterait aucune garantie sérieuse car il était livré à l'interprétation plus ou moins arbitraire des autorités du moment » et que la minorité avait pris « l'habitude de se résigner à la situation ${ }^{28}$ ". Les districts bilingues ne devaient donc pas être déterminés par des " décisions arbitraires d'un fonctionnaire, d'un ministre, d'un gouvernement ou même d'une assemblée législative particulière ${ }^{29}$ ». Il fallait les inscrire dans l'Acte de l'Amérique du Nord britannique ${ }^{30}$.

Leurs frontières devaient s'adapter à l'écoumène de la minorité francophone et non l'inverse ${ }^{31}$. En retraçant les frontières administratives à partir de cet écoumène, on pouvait « faire converger, au plan linguistique, des services émanant de compétences existantes" et permettre la collaboration entre le gouvernement fédéral et les provinces ${ }^{32}$. Aussi éviterait-on «l'extrême complexité de l'administration publique dans les pays à régime fédératif ", la multiplicité et l'enchevêtrement des aires de service et le découpage du territoire par de nombreux ministères, chacun dans leur sphère et pour mieux

${ }^{26}$ Commission BB, Rapport, vol. 1 : Les langues officielles, p. 89. En italique dans le texte.

27 Ibid., p. 97-98.

28 Ibid., p. 97.

29 Ibid., p. 74.

30 Ibid., p. 141.

31 Ibid., p. 120.

32 Ibid., p. 110, 115. 
desservir leurs propres fins administratives ${ }^{33}$. La Commission recommanda donc les divisions et subdivisions de recensement parce qu'elles étaient plus stables que les divisions administratives locales et qu'elles existaient, entre autres, pour " rendre compte de la composition linguistique du Canada ${ }^{34}$ ». Elle conserva 54 divisions de recensement qui respectaient le seuil des $10 \% 0^{35}$, mais refusa de les recommander comme districts bilingues initiaux. L'histoire montre que la Commission aurait dû recommander une première série de districts bilingues au lieu de déléguer cette tâche.

Le district bilingue n'était pas la solution idéale, mais elle était la meilleure pour quatre raisons. D'abord, il aurait imposé les plus importantes " modifications aux structures administratives ${ }^{36}$ ", $\mathrm{y}$ compris les structures provinciales et municipales : "Ce qui compte vraiment, ce n'est pas tant la reconnaissance d'un droit spécifique que la réorientation, sur le plan linguistique, d'un ensemble d'institutions relevant des trois niveaux de gouvernement ${ }^{37}$. " Deuxièmement, il n'aurait pas nécessité un bilinguisme institutionnel onéreux puisqu'il aurait été limité aux régions bilingues où " nos propositions aur[aient] les incidences les plus directes sur la vie des gens » au quotidien ${ }^{38}$. En troisième lieu, les gouvernements devaient pouvoir facilement y trouver le personnel bilingue nécessaire ${ }^{39}$. Enfin, déterminés à partir du seuil "raisonnable » de $10 \%{ }^{40}$, les districts n'auraient pas provoqué l'ire d'une majorité intolérante: «Dans ces régions, il sera[it] plus aisé, à l'ordinaire, de persuader la majorité de reconnaître la langue de la minorité que là où le fait minoritaire passe quasi inaperçu ${ }^{41}$. »

\footnotetext{
${ }^{33}$ Ibid., p. 115.

${ }^{34}$ Ibid., p. 111.

35 Ibid.

36 Ibid., p. 120.

37 Ibid., p. 109.

38 Ibid., p. 152.

39 Ibid., p. 110.

40 Ibid., p. 114.

${ }^{41}$ Ibid., p. 110.
} 
Ainsi, hormis sa reconnaissance symbolique de la minorité, sa transformation des structures administratives fait aussi du district bilingue la pierre angulaire du système. Les municipalités et les autres organismes publics locaux devaient permettre "l'emploi des deux langues officielles dans leurs délibérations » et publier leurs arrêtés et règlements en français et en anglais ${ }^{42}$. Les tribunaux devaient accepter des plaidoyers et fournir des services sténographiques et des services d'interprétation dans les deux langues ${ }^{43}$. Et surtout, les institutions fédérales et provinciales devaient traiter avec la population minoritaire locale dans sa langue, par écrit ou de vive voix. Il y a cinquante ans, de tels changements administratifs auraient été considérés comme révolutionnaires.

Les commissaires savaient que "la création des districts entraînera[it] d'importantes conséquences au plan administratif ${ }^{44}$ ". Par exemple, certaines villes situées tout près d'un district bilingue seraient exclues parce que leur population minoritaire francophone n'atteignait pas $10 \%{ }^{45}$. Et pourtant, c'est dans ces villes que se trouvent les bureaux gouvernementaux. Les commissaires proposeront des solutions contradictoires. D'un côté, il fallait « autant que possible former les districts de façon à n'y pas inclure des municipalités où la minorité n'atteint pas $10 \%{ }^{46}$ ». De l'autre, ces villes devaient faire " quand même partie du district pour profiter des services qui sont dispensés par les autres administrations locales et par les gouvernements provincial et fédéral ${ }^{47}$ ".

La Commission a également complexifié le concept en précisant que les services bilingues ne seraient pas les mêmes d'un district bilingue à l'autre. Il y aurait une gradation du bilinguisme institutionnel au pays : dans les institutions fédérales et dans les trois

\footnotetext{
42 Ibid., p. 117.

43 Ibid.

44 Ibid., p. 118.

45 Ibid.

46 Ibid., p. 116.

47 Ibid.
} 
provinces officiellement bilingues (Québec, Nouveau-Brunswick et Ontario), " tous les services " publics seraient offerts dans les deux langues dans les districts bilingues qui s'y trouvent ${ }^{48}$, mais seulement les "services essentiels" seraient offerts dans les districts bilingues situés ailleurs au pays ${ }^{49}$.

La commission BB voyait donc d'un bon œil les régions bilingues établies en 1938 et améliorées par la Commission du service civil en 1967, suite à la commission Glassco. Elle savait qu'on pouvait difficilement chambarder une bureaucratie si lourde et qu'il était inutile de proposer un bilinguisme sans limite a mari usque ad mare $e^{50}$. La réforme de l'appareil fédéral devait donc respecter « le réalisme le plus élémentaire ${ }^{51}$ ", c'est-à-dire que le bilinguisme devait se limiter aux régions du pays où la minorité formait une masse critique. Néanmoins, elle n'était pas satisfaite de l'approche bureaucratique et discrétionnaire qui soutenait ces régions bilingues.

Sept recommandations portaient sur les districts bilingues. La Commission n'a pas proposé de districts bilingues; elle a plutôt demandé aux gouvernements du Canada et des provinces d'établir des districts conjoints ou particuliers et un conseil conjoint pour réviser les districts bilingues initiaux afin d'en ajouter ou d'en éliminer, selon les données de chaque recensement. Une autre recommandation portait sur la capitale nationale comme district bilingue particulier. Les autres recommandations afférentes aux districts bilingues traitaient des obligations provinciales en matière de services publics dans la langue minoritaire, notamment en éducation.

\footnotetext{
48 Ibid., p. 121.

49 Ibid., p. 122.

50 Ibid., p. XxxiII.

51 Ibid.
} 


\section{Les structures administratives fédérales}

Plusieurs autres recommandations concernaient la restructuration de l'appareil fédéral pour assurer l'égalité des deux communautés linguistiques. Ces recommandations structurelles sont de deux types. Certaines visaient quatre nouvelles unités administratives - les unités francophones, le Commissariat aux langues officielles, la capitale fédérale et l'Office des langues de la fonction publique - et d'autres se rapportaient à l'intégration du bilinguisme dans l'appareil fédéral.

Puisque cet appareil était un bastion anglophone, elle a proposé la création d'unités administratives francophones pour permettre aux francophones de gravir les échelons bureaucratiques en français sans s'assimiler. L'unité francophone devait être "un principe fondamental d'organisation et de gestion " au sein de l'appareil fédéral ${ }^{52}$. Toutes les institutions fédérales devaient établir des unités francophones pour garantir que «le français soit langue de travail ${ }^{53}$ ".

Le Commissaire aux langues officielles, pour sa part, serait chargé de veiller au respect du statut du français et de l'anglais au Canada. Cela comprenait les droits linguistiques des fonctionnaires.

La capitale fédérale devait être désignée district bilingue particulier. Le gouvernement fédéral devait également y " promouvoir, sous tous ses aspects, l'égalité entre anglophones et francophones $^{54}$ ", notamment en assumant les coûts des panneaux extérieurs et intérieurs de tous les immeubles appartenant au Gouvernement du Canada ou loués par lui. Ottawa devait aussi créer, en partenariat avec le Québec et l'Ontario, un "organisme consultatif tripartite " chargé de la mise en ouvre des recommandations de la Commission pour la capitale nationale ${ }^{55}$.

52 Commission BB, Rapport, vol. 3 : Le monde du travail, Ottawa, Imprimeur de la reine, 1969 , p. 279 . En gras dans le texte.

53 Ibid.

${ }^{54}$ Commission BB, Rapport, vol. 5 : La capitale fédérale, Ottawa, Imprimeur de la reine, 1970, p. 53. En gras dans le texte.

55 Ibid., p. 88. En gras dans le texte. 
La Commission a aussi proposé la création d'un Office des langues de la fonction publique pour " concevoir, mettre en œuvre et maintenir le bilinguisme institutionnel ", " jouer le rôle d'un guide du gouvernement dans son ensemble, et d'un animateur auprès [... des] ministères, sociétés de la Couronne et autres organismes fédéraux ", " coordonner, faciliter et contrôler l'activité des services linguistiques des ministères ", " définir la place et le rôle de la traduction » et assurer et évaluer " la recherche sur le programme du bilinguisme institutionnel ${ }^{56}$ ».

En second lieu, le bilinguisme allait faire partie de la culture organisationnelle au fédéral. Le gouvernement devait dorénavant nommer les sous-ministres, sous-ministres associés, sous-ministres adjoints et leurs équivalents dans les autres organismes fédéraux de façon à assurer "l'équilibre des rôles entre francophones et anglophones $^{57}$ ». Il fallait aussi établir, au sein des institutions fédérales, " un service linguistique " relevant directement du sousministre afin de " concevoir, mettre en œuvre et maintenir un régime de bilinguisme institutionnel, et de remplir, au sein du ministère, les fonctions attribuées à l'Office des langues de la fonction publique ${ }^{58}$ ". Il fallait aussi transformer les Forces armées en une institution bilingue. Cela comprenait deux collèges militaires - un collège anglophone à Kingston et un collège francophone à Saint-Jean-sur-Richelieu - ainsi que l'enseignement des deux langues officielles aux officiers ${ }^{59}$.

\section{Le soutien fédéral à l'éducation en milieu minoritaire}

La Commission a débordé les limites de son mandat en s'immisçant dans des responsabilités provinciales car elle croyait que "négliger l'action des gouvernements provinciaux et locaux, ce serait proposer

56 Commission BB, Rapport, vol. 3 : Le monde du travail, p. 300. En gras dans le texte.

57 Ibid., p. 286. En gras dans le texte.

58 Ibid., p. 301.

59 Ibid., p. 356. 
des moyens valables mais incomplets ${ }^{60}$ ". C'est notamment le cas en éducation parce que l'école est " le cadre le plus nécessaire au maintien de la langue et de la culture; celles-ci, à défaut d'école, ne peuvent conserver leur vitalité61 ". Puisque les systèmes scolaires étaient conçus " en fonction de la majorité linguistique dans les provinces anglophones ", la minorité devait obtenir un "régime d'enseignement conforme à ses yeux ", des écoles distinctes comme « contrepoids » à l'ambiance anglophone et un « programme scolaire [pour] refléter ces différences" culturelles ${ }^{62}$.

Sachant que les provinces garderaient jalousement ce domaine et hésiteraient à intervenir, la Commission a recommandé de l'aide financière de la part du gouvernement fédéral. Ainsi, Ottawa devait prendre à sa charge les dépenses supplémentaires qu'entraînent l'enseignement dans la langue minoritaire et la formation des enseignants ${ }^{63}$. Le gouvernement fédéral devait également fournir une allocation aux étudiants qui poursuivaient leurs études universitaires en français en dehors de leur province ainsi qu'un octroi aux provinces pour soutenir leurs universités de langue française ${ }^{64}$.

\section{Le soutien fédéral aux provinces et aux municipalités}

La Commission reconnaissait que le Gouvernement du Canada était mieux nanti et qu'il devait jouer un rôle prépondérant en matière de bilinguisme et de biculturalisme à tous les niveaux de la société canadienne. Elle a donc recommandé que le Gouvernement du Canada accorde des subventions aux municipalités ontariennes et québécoises faisant partie de la capitale nationale afin d'y promouvoir les langues officielles ${ }^{65}$ et qu'il collabore avec le Gouvernement du

${ }^{60}$ Commission BB, Rapport, vol. 1 : Les langues officielles, p. 92.

${ }^{61}$ Ibid., p. 128.

${ }^{62}$ Commission BB, Rapport, vol. 2 : L'éducation, 1968, p. 8-10.

63 Ibid., p. 201-203.

64 Ibid., p. 192-194.

${ }^{65}$ Commission BB, Rapport, vol. 5 : La capitale fédérale, p. 60. 
Québec pour faire du français la «principale langue de travail au Québec »dans le secteur privé ${ }^{66}$.

\section{Les mesures administratives internes}

Dans sa première recommandation, la Commission proposa que " l'anglais et le français soient formellement déclarés langues officielles du Parlement du Canada, des tribunaux fédéraux, du gouvernement fédéral et de l'administration fédérale ${ }^{67}$ ". Son rapport sur le monde du travail recommanda «l'équilibre des rôles entre anglophones et francophones "dans tous les organismes fédéraux de planification et de consultation ${ }^{68}$. Il fallait que les avis, directives, formulaires et manuels soient diffusés dans les deux langues officielles ${ }^{69}$. Aussi proposa-t-elle un amendement à la Loi sur l'emploi dans la fonction publique ainsi qu'aux règlements afférents et aux conventions collectives afin d'assurer que les communications entre employeur et employés "s'effectuent en anglais ou en français au choix de l'employé ${ }^{70}$ ». Elle préconisait la classification des postes suivant leurs exigences linguistiques ${ }^{71}$. D'autres recommandations portaient sur l'amélioration de la formation et de la compétence linguistique des fonctionnaires fédéraux ${ }^{72}$. La Commission a aussi recommandé la fin immédiate de la rédaction des textes en anglais, qui devaient ensuite être traduits en français ${ }^{73}$. Elle a également proposé plusieurs mesures pour rendre bilingues les communications entre le gouvernement fédéral et les entreprises, surtout en offrant la version française des documents, tels les contrats et les appels

66 Commission BB, Rapport, vol. 3 : Le monde du travail, p. 579.

67 Commission BB, Rapport, vol. 1 : Les langues officielles, p. 94. En gras dans le texte.

${ }^{68}$ Commission BB, Rapport, vol. 3 : Le monde du travail, p. 286-287.

69 Ibid., p. 289.

70 Ibid., p. 290.

${ }^{71}$ Ibid., p. 291.

72 Ibid., p. 293.

73 Ibid., p. 294. 
d'offre ${ }^{74}$, et pour assurer que le français soit une langue de travail quotidienne dans les Forces armées ${ }^{75}$.

\section{La mise en œuvre des recommandations}

Le gouvernement fédéral a bien tenté de mettre en œuvre les 109 recommandations, y compris celles portant sur les districts bilingues et les unités de langue française. Néanmoins, il n’a pas réussi en raison, notamment, de quelques recommandations qui se sont révélées problématiques. Le bilan est toutefois positif. Les fonctionnaires ont joué un rôle déterminant dans le succès et les échecs du gouvernement.

\section{Les districts bilingues}

Concernant les districts bilingues, la réaction initiale des hauts fonctionnaires au Conseil privé fut mitigée $\mathrm{e}^{76}$. Selon eux, les divisions et subdivisions du recensement n'étaient pas appropriées. Les régions bilingues, telles que dénombrées en 1967, pouvaient atteindre l'objectif moyennant quelques révisions. Ils acceptent d'éliminer le seuil des 10000 habitants et suggèrent d'appliquer celui des $10 \%$ avec flexibilité pour créer davantage de districts bilingues. Aussi rejettent-ils la recommandation de la Commission d'éliminer un district bilingue si la population minoritaire tombe sous le seuil des $7 \%$. Ainsi, même si la minorité ne forme plus $10 \%$ de la population, une région devrait demeurer un district bilingue. Enfin, ils souhaitent que le Cabinet Pearson adopte une formule de rechange au district bilingue pour desservir les grands centres urbains où la minorité ne forme pas $10 \%$ des habitants. Ils sont donc prêts à aller plus loin que la Commission à plusieurs égards.

Ces hauts fonctionnaires jouent également un rôle positif lors de la rédaction de la Loi sur les langues officielles. Face au refus des

74 Ibid., p. 593-594.

75 Ibid., p. 347-356.

${ }^{76}$ Memorandum to the Cabinet, Cabinet document 13/1968, Ottawa, 10 janvier 1968. 
provinces de créer des districts bilingues conjoints, ils permettent la création de districts " fédéraux ». Ils favorisent aussi l'établissement de districts conjoints plus tard, si les provinces se ravisaient, en demandant de tracer les frontières des districts fédéraux de façon à faciliter une superposition éventuelle aux districts provinciaux. Aussi refusent-ils de se limiter à l'écoumène de la minorité francophone défini selon les unités de recensement recommandées par la Commission; ils ajoutent comme unités additionnelles une municipalité, un district scolaire, une circonscription électorale fédérale et une circonscription électorale provinciale.

En revanche, les hauts fonctionnaires limitent les services bilingues à ceux qui sont destinés au " public " et offerts par les «bureaux principaux ». D'une part, les services de laboratoire, par exemple, ne seraient pas tenus de fournir les résultats de leurs analyses dans les deux langues s'ils ne traitent pas directement avec le public. D'autre part, les bureaux secondaires, situés dans les districts bilingues, et les bureaux principaux, situés à l'extérieur d'un district bilingue, n'auraient pas à fournir de services dans les deux langues. Cette limite respecte les règlements adoptés par la Commission du service civil en 1967 selon lesquels les services bilingues seront fournis par l'unité administrative située dans les 82 régions bilingues. Enfin, le projet de loi rédigé par ces hauts fonctionnaires reste muet sur la valeur symbolique du district bilingue.

Les hauts fonctionnaires fédéraux exerceront aussi une influence lors de la désignation des districts bilingues menée par les deux conseils consultatifs nommés à cette fin. Leur réaction au rapport du premier comité consultatif est très positive. Ils recommandent au gouvernement Trudeau de "procéder à la création des districts bilingues en s'inspirant des recommandations du Conseil " et "à une date aussi rapprochée que possible ${ }^{77}$ ». Ils rejettent même l'éventualité d'attendre les données de 1971. Or les tergiversations du ministre responsable

77 Rapport du Conseil consultatif des districts bilingues, Mémoire au Cabinet du Secrétaire d'État, Document du Cabinet 414-71, Ottawa, 14 avril 1971. 
du dossier, Gérard Pelletier, concernant la validité des données de 1961 comme fondement des premiers districts bilingues poussent ces hauts fonctionnaires à se pencher plus en détail sur ce défi. Finalement, ils recommandent que " le Cabinet donne son approbation à la création des districts bilingues fédéraux " aussitôt que possible, tout en élevant le seuil à $20 \%$ afin d'éviter des « controverses politiques " au premier tour ${ }^{78}$, notamment celles pouvant découler de la recommandation du comité consultatif de faire de tout le Québec un district bilingue.

Un an après la réception du rapport du premier Conseil consultatif, les hauts fonctionnaires font volte-face. Ils constatent des " changements considérables " dans les données du recensement de 1971 relativement aux 28 districts bilingues recommandés selon le seuil des $20 \%{ }^{79}$. Pourtant, le recensement indique qu'un seul des 28 districts bilingues ne correspond pas à ce seuil. Le Cabinet Trudeau décide néanmoins d'établir un second Conseil consultatif et d'attendre son rapport.

Les hauts fonctionnaires influenceront le travail du second Conseil consultatif. Le premier avait consulté les députés fédéraux et provinciaux et les citoyens majoritaires et minoritaires dans les districts potentiels ainsi que tous les gouvernements provinciaux. Le second en fait autant, mais il consulte également les hauts fonctionnaires du Conseil du trésor, à la demande de ceux-ci, pour comprendre les progrès accomplis quant aux régions bilingues, en attendant la création de districts bilingues, et quant à l'application du principe de l'importance de la demande à l'extérieur des districts bilingues. Ces hauts fonctionnaires manifestaient de l'inquiétude au sujet du travail du Conseil consultatif, craignant que ses recommandations rendent désuètes ces régions bilingues ${ }^{80}$.

${ }^{78}$ Recommandations du Conseil consultatif des districts bilingues, Mémoire au Cabinet, Secrétariat d'État, Document du Cabinet 832-71, Ottawa, 14 juillet 1971.

79 Cabinet Minutes, 11 mai 1972, Ottawa, Privy Council, Document 19-72, 1972, p. 5.

80 " Procès-verbal de la $13^{\text {e }}$ Réunion, les 12,13 et 14 octobre 1973 ", Ottawa, Conseil consultatif des districts bilingues, 1973, p. 3. 
Jusqu'en février 1972, le Secrétariat d'État était responsable des langues officielles, alors que le Conseil du trésor et la Commission de la fonction publique étaient responsables de la mise en œuvre des mesures administratives. Or, conscient qu'il ne pouvait imposer de lignes directrices aux autres ministères, Gérard Pelletier demanda au Cabinet de déléguer ce pouvoir au Conseil du trésor, tout en conservant la responsabilité des districts bilingues. Le Conseil du trésor avait déjà reçu le pouvoir de déterminer les postes bilingues à pourvoir dans les districts bilingues et de fixer les exigences linguistiques de ces postes. C'est à partir de mars 1971 que le Conseil du trésor lance son système de prestation de services bilingues dans les « régions bilingues ».

Deux ans plus tard, le Conseil du trésor met en place son système, en émettant une directive - la circulaire 1973-88 comment il désignera et comblera les postes bilingues dans les districts bilingues et comment il déterminera l'importance de la demande en dehors des districts bilingues, soit dans les quartiers généraux des institutions fédérales, les bureaux desservant le public voyageur et les bureaux fédéraux situés à l'étranger. La directive définit aussi les « régions bilingues » - certaines parties de Montréal, du Québec, du nord et de l'est de l'Ontario et du Nouveau-Brunswick - et ajoute que les fonctionnaires travaillant dans ces régions bilingues doivent être bilingues. Elle précise les types d'occupation et le niveau des postes bilingues requis dans ces régions bilingues.

Lorsque les gestionnaires rencontrent les membres du Conseil consultatif en octobre 1973, les dispositions de la circulaire n'étaient pas encore entrées en vigueur. D'où l'importance de la rencontre. Or la plupart des membres du Conseil consultatif accordent peu d'importance à ces « considérations administratives » et expriment la crainte

${ }^{81}$ Circulaire 1973-88 : exigences linguistiques des postes, Ottawa, Conseil du trésor, 29 juin 1973. 
que ces hauts fonctionnaires tentent de remplacer les districts bilingues ${ }^{82}$.

La bureaucratie fédérale a exercé plus d'influence sur le travail du Conseil consultatif dans son interprétation des concepts statutaires. En fait, le Conseil consultatif a du mal à interpréter les expressions " principaux bureaux » et " demande importante ». Il doit demander des précisions aux fonctionnaires. La commission BB avait évoqué ces défis et proposé des solutions ${ }^{83}$, mais elle n'avait pas poussé sa réflexion plus loin. Pour clarifier la différence entre le premier paragraphe de l'article 9, portant sur les districts bilingues, et le second, portant sur la " demande importante " à l'extérieur des districts bilingues, concept qui a mené à la mise à jour des régions bilingues, les membres du Conseil demandent l'avis d'un haut fonctionnaire du ministère de la Justice. Ce dernier déclare d'abord que le Conseil n'a rien à voir avec le second paragraphe, puis affirme que ce second paragraphe remplacera éventuellement le premier, mais admet par la suite que le second accorde trop de latitude au Conseil du trésor. Les membres du Conseil consultatif demeurent confus.

Jusqu'à la publication du rapport du second Conseil consultatif, en novembre 1975, l'influence de la bureaucratie fédérale sur les districts bilingues fut mitigée. Par la suite, cette influence sera déterminante et ce seront, en fait, les dirigeants du Conseil du trésor qui saboteront la pierre angulaire du projet. La commission BB se méfiait du Conseil du trésor, même si elle avait proposé qu’on lui confie le mandat de mettre en œuvre le bilinguisme institutionnel fédéral ${ }^{84}$, parce que cet organisme central détenait l'autorité nécessaire. En effet, elle avait pris bien soin de préciser que le Conseil du trésor ne devait pas déterminer les districts bilingues parce que ses dirigeants étaient trop bureaucratiques et trop peu habiles pour s'acquitter

82 Daniel Bourgeois, La genèse, la spécification et l'abandon des districts bilingues canadiens, 1966-1976, thèse de doctorat (science politique), Québec, Université Laval, avril 1997, p. 198.

${ }^{83}$ Commission BB, Rapport, vol. 1 : Les langues officielles, p. 118-120.

${ }^{84}$ Commission BB, Rapport, vol. 3 : Le monde du travail, p. 302-303. 
adéquatement d'une tâche politique si délicate ${ }^{85}$. L'histoire montre que la Commission avait raison.

Toutefois, la réaction initiale des hauts fonctionnaires du Bureau du Conseil privé au rapport du second Conseil consultatif fut positive. Ils recommandèrent au Cabinet de retenir l'idée du district bilingue et jugèrent " acceptables » les 30 districts proposés ${ }^{86}$. Le gouvernement promit alors de procéder " à la proclamation de districts bilingues " dès que possible ${ }^{87}$.

Une première étude du rapport du Conseil consultatif, réalisée par des fonctionnaires du Conseil du trésor, recommanda la création de 31 districts bilingues semblables à ceux qu'avait proposé le second Conseil consultatif ${ }^{88}$. Le président du Conseil du trésor, Jean Chrétien, accepta de soumettre le rapport au Cabinet. Toutefois, les nouveaux hauts fonctionnaires au Conseil du trésor, ignorant la valeur symbolique des districts bilingues et constatant que leur proclamation chambarderait leurs régions bilingues, décidèrent, en mai 1976, à l'insu du président, de soumettre un autre rapport au Cabinet ${ }^{89}$. Ce nouveau rapport recommandait que le gouvernement « ne procède pas à l'établissement de districts bilingues » et que, plutôt, il « réaffirme sa politique relative à la fourniture de services bilingues adéquats dans l'ensemble du pays » en fonction du système des régions bilingues établies, depuis 1973, selon l'importance de la demande ${ }^{90}$. Ces deux

85 Ibid., p. 285.

86 Rapport du second Conseil consultatif des districts bilingues, Document 630-75, 7 novembre 1975.

87 Charles M. Drury, "Déclaration ", 21 novembre 1975, dans Canada, Débats de la Chambre des communes : compte rendu offciel, $30^{\circ}$ Législature, $1^{\text {re }}$ session, vol. 9 : 1975 : du 31 octobre 1975 au 9 décembre 1975, inclusivement, Ottawa, Imprimeur de la reine, p. 9327, [En ligne], [http://parl.canadiana.ca/ view/oop.debates_CDC3001_09/593?r=0\&s=1], image 593.

88 Implementation of the Official Languages Act with respect to the establishment of Bilingual Districts and related measures, Ottawa, Treasury Board, 25 mai 1976.

89 Bourgeois, Canadian Bilingual Districts, p. 191.

90 Memorandum to Cabinet: Bilingual Districts and Related Measures, Ottawa, Treasury Board, septembre 1976. 
hauts fonctionnaires ont ainsi contrecarré la mise en place des districts bilingues six mois avant le Cabinet.

Les districts bilingues recommandés par la commission BB devaient remplacer les régions bilingues établies par l'appareil fédéral en 1938 et peaufinées en 1966 et 1973, en accordant une grande valeur symbolique à la " districtisation " administrative tripartite. Finalement, ce sont plutôt les régions bilingues bureaucratiques qui se sont imposées sur les districts bilingues symboliques.

La commission BB n'appréciait peut-être pas à juste titre la complexité administrative d'une "districtisation » bilingue, comme en témoigne la complexité des règlements adoptés en 1992 pour préciser les régions bilingues ${ }^{91}$. Mais c’est justement la simplicité du concept de district bilingue qui lui donne sa valeur ajoutée. Les districts bilingues devaient revigorer les communautés francophones hors Québec et limiter l'usage de l'anglais dans cette province. Ils devaient changer les appareils fédéral, provincial et municipal en les adaptant à l'écoumène minoritaire dans le but de sauver le pays. Les gestionnaires du Conseil du trésor n’ont pas su apprécier leur valeur symbolique et l'importance d'inciter les provinces et les municipalités à suivre l'exemple. Ils craignaient que leurs efforts pour établir des régions bilingues selon l'importance de la demande soient annihilés par l'adoption de districts bilingues. Ils ont donc procédé à une "déviation bureaucratique ${ }^{92}$ ".

La déviation orchestrée par les dirigeants du Conseil du trésor ne représente toutefois pas l'opinion de tous au sein de l'appareil fédéral. Plusieurs hauts fonctionnaires du Bureau du Conseil privé partagent la crainte de la commission $\mathrm{BB}$ concernant la rigidité bureaucratique du Conseil du trésor. Ils redoutent que ses dirigeants n'usurpent le pouvoir des comités du Cabinet en matière de langues officielles et sabotent les efforts du comité interministériel qui a produit

91 Règlements sur les langues officielles : communications avec le public et prestation des services, dans Gazette du Canada, partie II, vol. 126, nº 1, $1^{\mathrm{er}}$ janvier 1992.

92 Bourgeois, La genèse, la spécification et l'abandon des districts bilingues canadiens, 1966-1976, p. 352. 
le rapport approuvé par son président ${ }^{93}$. Ils craignent cette déviation bureaucratique $^{94}$ et se doutent que ces hauts dirigeants ne comprennent pas la valeur symbolique des districts bilingues ${ }^{95}$. Leurs avertissements ne semblent toutefois pas s'être rendus jusqu'au Cabinet.

La commission BB avait raison de se méfier des bureaucrates du Conseil du trésor, mais on ne saura jamais si elle a eu raison de chambouler l'appareil fédéral avec des districts bilingues. En effet, aucun district bilingue n'a vu le jour. Il est donc impossible de procéder à l'évaluation de cette pierre angulaire du bilinguisme canadien. Néanmoins, l'absence de leur valeur symbolique se fait sentir ${ }^{96}$. Les régions bilingues, établies en 1938 et mises à jour en 1966, 1973 et 1992 , sont peu connues et très complexes. Certains services de certaines institutions sont offerts dans certaines régions alors que davantage de services de plusieurs autres institutions sont fournis dans d'autres régions. De plus, peu de Canadiens savent quels services sont disponibles dans les deux langues et à quels endroits au pays. Par ailleurs, même s'il est enfin possible de superposer les régions bilingues fédérales et provinciales ${ }^{97}$, le Conseil du trésor n'a fait aucun effort en ce sens.

\section{Les structures administratives fédérales}

Si le gouvernement fédéral a étouffé dans l'œuf la création des districts bilingues, il a au moins mis sur pied des unités francophones. Un projet pilote a créé, en 1971, 475 unités, qui emploient 29000 fonctionnaires ${ }^{98}$. Cette expérience a produit deux types d'échec ${ }^{99}$ :

93 Clovis Demers, « Note à Monsieur Ross - Districts bilingues », Bureau du Conseil privé, Ottawa, 29 juin 1976.

${ }_{94}$ Memorandum for Mr. Joncas, J. S. Hodgson, Privy Council Office, $1^{\mathrm{er}}$ septembre 1976.

95 Memorandum to Cabinet: Bilingual Districts and Related Measures.

96 Bourgeois, Canadian Bilingual Districts, p. 271.

${ }^{97}$ Ibid., p. 265.

${ }^{98}$ Circulaire 1970-95 : unités de langue française, Ottawa, Conseil du trésor, 14 septembre 1970.

99 V. Seymour Wilson, «Language Policy », dans G. Bruce Doern et V. Seymour Wilson (dir.), Issues in Canadian Public Policy, Toronto, Macmillan, 1974, p. 253-285. 
d'abord, plusieurs francophones ont craint que ces unités ne limitent leur avancement professionnel en réduisant les occasions d'apprendre l'anglais; ensuite, plusieurs francophones ont affirmé perdre du temps à accompagner leurs collègues anglophones qui se sont joints à l'unité pour apprendre le français ${ }^{100}$. Le Conseil du trésor a ressuscité l'initiative en $1975^{101}$, mais en vain ${ }^{102}$. On n'en a plus jamais entendu parler.

La Commission avait de bonnes intentions, mais le regroupement des fonctionnaires en unités distinctes semble une mesure trop poussée, inefficace et peu appréciée, notamment par les francophones. Elle semblait nécessaire au milieu des années 1960 alors que l'appareil fédéral était un bastion anglophone, mais la Loi sur les langues officielles de 1969 et les autres mesures gouvernementales prises en 1966 et 1967 ont changé la donne. Néanmoins, les rapports annuels du Commissaire aux langues officielles révèlent toujours que les fonctionnaires francophones ont peine à travailler en français, même depuis l'insertion de ce droit en 1988 dans la nouvelle Loi sur les langues officielles. Toutefois, personne ne revendique la création d'unités de langue française.

\section{Le soutien fédéral à l'éducation en milieu minoritaire}

Dès 1969, le Secrétariat d'État lance un programme de soutien financier aux provinces et aux territoires dans le but de favoriser l'éducation en milieu minoritaire, connu de nos jours sous le nom de Programme des langues officielles en éducation. Ce programme

100 René Matte, «Déclaration ", 23 juin 1970, dans Canada, Compte rendu officiel des débats de la Chambre des communes, 28e Législature, $2^{e}$ session, vol. $8: 1970:$ du 5 juin au 7 octobre, Ottawa, Imprimeur de la reine, 1970, p. 8494, [En ligne], [http:// parl.canadiana.ca/view/oop.debates_CDC2802_08/732?r=0\&s=2], image 732.

101 Circulaire 1975-11 : unités de travail en français, Ottawa, Conseil du trésor, 25 septembre 1975 .

102 Jacques Robichaud, «Le bilinguisme dans l'administration fédérale du Canada (1969-1982) ", Les Cahiers de droit, vol. 24, n 1 (1983), p. 115-130, [En ligne], [http://id.erudit.org/iderudit/042537ar]. 
s'occupe surtout d'éducation dans la langue minoritaire comme langue maternelle, mais également des programmes d'immersion ${ }^{103}$.

Toutefois, la plus grande contribution de la Commission en matière d'éducation en milieu minoritaire a été sa recommandation d'insérer dans l'Acte de l'Amérique du Nord britannique le droit de la minorité francophone hors Québec à des écoles distinctes et à la gestion de celles-ci. Cette recommandation a mené à l'article 23 de la Charte canadienne des droits et libertés ${ }^{104}$.

En fait, la Commission préconisait « une administration particulière et de caractère propre répondant aux besoins de la minorité » en éducation ${ }^{105}$, mais elle a refusé de recommander la formation de conseils scolaires minoritaires afin de permettre aux minorités francophones de tirer profit de la centralisation en éducation, en vogue dans les années $1960^{106}$. La Commission montrait ainsi une bonne compréhension des tendances administratives et des avantages de la centralisation.

Malgré le refus de la Commission de recommander la gestion scolaire minoritaire, ses écrits ont ironiquement mené à ce résultat. En effet, citant les rapports de la Commission, la Cour suprême du Canada a donné suite de deux façons à l'expression « des établissements d'enseignement de la minorité linguistique ", contenue dans l'article $23^{107}$. D’une part, la Cour suprême a décidé que la minorité devait

103 Matthew Hayday, Bilingual Today, United Tomorrow: Official Languages in Education and Canadian Federalism, Montréal, McGill-Queen's University Press, 2005.

104 Daniel Bourgeois, "La gestion scolaire, pierre angulaire des minorités linguistiques du Canada ", dans Jules Rocque (dir.), La direction d'école et le leadership pédagogique en milieu francophone minoritaire : considérations théoriques pour une pratique éclairée, avec une préface de Michel Bastarache et une postface de Jean-Joseph Moisset, Winnipeg, Presses universitaires de Saint-Boniface, 2011, p. 101-119.

105 Commission BB, Rapport, vol. 2 : L'éducation, p. 171.

106 Daniel Bourgeois, Vers la pleine gestion scolaire francophone en milieu minoritaire: étude des besoins des trente conseils scolaires francophones en milieu minoritaire au Canada, Moncton, Institut canadien de recherche sur les minorités linguistiques et Fédération nationale des conseils scolaires francophones, 2004.

107 Charte canadienne des droits et libertés (Annexe B : Loi de 1982 sur le Canada), 31 Elizabeth II, chap. 11. 
" avoir à l'égard des établissements d'enseignement une certaine mesure de gestion et de contrôle ${ }^{108}$ ». D'autre part, elle a imposé aux provinces et aux territoires " des obligations positives de changer ou de créer d'importantes structures institutionnelles ${ }^{109}$ ". Depuis, des conseils scolaires minoritaires desservent l'ensemble des minorités à l'échelle du pays. Selon certains, la minorité francophone exerce la pleine gestion scolaire de nos jours ${ }^{110}$.

\section{Le soutien fédéral aux provinces}

La Commission portait sur des questions d'ordre national, mais elle savait qu'elle ne pouvait négliger l'apport des gouvernements provinciaux, surtout dans le domaine de l'éducation. Ottawa a relevé le défi posé par la Commission en suivant presque à la lettre toutes ses recommandations. Le gouvernement fédéral a encouragé les provinces et les territoires à promouvoir les langues officielles et à mieux desservir leur minorité de langue officielle. Les provinces et les territoires ont depuis adopté plusieurs mesures favorables aux minorités francophones, en grande partie grâce à l'appui du gouvernement fédéral ${ }^{111}$. Les municipalités ont également suivi l'exemple ${ }^{112}$.

108 Mahéc. Alberta (1990), 1 R.C.S., 342, p. 370, [En ligne], [http://scc-csc.lexum.com/ scc-csc/scc-csc/fr/item/580/index.do].

109 Ibid., p. 365.

110 Michael D. Behiels, Canada's Francophone Minority Communities: Constitutional Renewal and the Winning of School Governance, Montréal, McGill-Queen's University Press, 2004. Nous avons montré le contraire dans Bourgeois, Vers la pleine gestion scolaire francophone en milieu minoritaire.

111 Daniel Bourgeois et al., La contribution des gouvernements provinciaux et territoriaux à l'épanouissement des communautés francophones en milieu minoritaire : un premier bilan et quelques prospectives, Moncton, Institut canadien de recherche sur les minorités linguistiques, 2007.

112 Daniel Bourgeois et Yves Bourgeois, «Les municipalités canadiennes et les langues officielles ", Revue canadienne de science politique, vol. 44, nº 4 (décembre 2011), p. 789-806, [En ligne], [http://dx.doi.org/10.1017/S0008423911000758]. 
Le bilan est toutefois plus mitigé en ce qui concerne la minorité anglophone du Québec ${ }^{113}$. La Commission souhaitait un Québec davantage français, mais voulait également que la position enviable de l'anglais dans cette province serve d'exemple dans l'épanouissement du français à l'extérieur du Québec. La Charte de la langue française de 1977 affirme la primauté du français au Québec, mais son adoption visait, en partie, à contrer le bilinguisme officiel fédéral et à réduire le bilinguisme provincial. La juxtaposition des deux objectifs poursuivis par la Commission a donc mené à un Québec plus français, mais aussi à la disparition de l'exemple québécois.

\section{Les mesures administratives internes}

Ottawa a mis en œuvre les mesures administratives proposées par la Commission. On retrouve un certain équilibre pour ce qui est des rôles entre anglophones et francophones au sein de la hiérarchie fédérale. Les avis, directives, formulaires et manuels sont publiés dans les deux langues. Les communications entre employés et employeur s'effectuent dans la langue des premiers. La plupart des postes comportent des exigences linguistiques. Malgré des lacunes ${ }^{114}$, la formation linguistique a permis à plusieurs fonctionnaires d'acquérir l'autre langue officielle. Le français est maintenant langue de travail au sein des Forces armées. Les contrats, appels d'offre et autres documents ciblant le secteur privé sont publiés en français et en anglais. On n'a pas créé l'Office des langues de la fonction publique, mais des responsables des langues officielles jouent ce rôle au sein de chaque institution. L'égalité réelle n'est pas atteinte - par exemple, le français est encore une langue de traduction plutôt que de

113 Richard Bourhis, The Vitality of the English-Speaking Communities of Quebec: From Community Decline to Revival, Moncton, Institut canadien de recherche sur les minorités linguistiques, 2008.

114 Gilles Bibeau, Rapport de l'étude indépendante sur les programmes de formation linguistique de la Fonction publique du Canada, Ottawa, Conseil du trésor, 1976. 
rédaction, même dans les régions bilingues ${ }^{115}$ - mais " l'usine " ne ressemble pas à celle que Laurendeau critiquait en 1962.

\section{Analyse}

Notre lecture des rapports et procès-verbaux de la Commission et nos entrevues avec plus de 20 employés et hauts gestionnaires impliqués dans le dossier nous mènent à deux conclusions. La première est normative : la Commission avait une bonne compréhension de l'appareil fédéral. La seconde est scientifique : la structuration de la mise en œuvre des mesures administratives a contribué à l'échec de certaines mesures, notamment la création des districts bilingues.

\section{La Commission avait une bonne compréhension de l'appareil fédéral}

La Commission était à l'affût des tendances administratives. Elle n'a pas proposé l'autogestion scolaire en milieu minoritaire pour que les minorités bénéficient de la gestion de l'éducation par les gouvernements provinciaux. Par contre, sa connaissance de l'autogestion et des nationalismes en vogue l'a poussée à proposer que la minorité puisse prendre des décisions en matière d'éducation. Elle a aussi prévu l'ascension du Conseil du trésor comme puissant organisme central et technocrate.

Toutefois, certaines recommandations se sont révélées problématiques. C'est le cas des districts bilingues, des unités de langue française et de l'Office des langues de la fonction publique. Ces mesures semblaient trop radicales pour l'appareil fédéral. Or la Commission pensait que des mesures extraordinaires étaient requises pour maintenir l'unité du Canada. Rappelons que les mesures administratives discrétionnaires mises en place antérieurement avaient alimenté le mouvement séparatiste et la crise de l'unité nationale, et

115 Matthieu LeBlanc, "Pratiques langagières dans un milieu de travail bilingue de Moncton ", Francophonies d'Amérique, no 22 (automne 2006), p. 121-139, [En ligne], [http://id.erudit.org/iderudit/1005382ar]. 
poussé le gouvernement Pearson à instituer une commission royale d'enquête.

L'appareil fédéral aurait-il mis en œuvre les 109 mesures proposées par la Commission sans l'apport de celle-ci? Certes, la déclaration de Therrien a poussé le gouvernement à adopter plusieurs mesures qu'on retrouvera dans les rapports de la Commission, y compris la "districtisation » du bilinguisme, mais la Commission a joué un rôle fondamental en la matière, en fournissant les résultats des recherches et des arguments d'ordre sociologique pour justifier et soutenir les mesures mises en œuvre.

Le cas des districts bilingues est révélateur du rôle joué par la Commission et des limites de l'appareil d'État. La pierre angulaire du système, les districts bilingues, aurait pu facilement être calquée sur les 82 régions bilingues évoquées en février 1966 selon le seuil de $10 \%$ et le nombre de 10000 habitants vivants en milieu minoritaire. Les fonctionnaires auraient pu recommander au Cabinet de transformer ces 82 régions en districts bilingues, dans le prolongement des recommandations de la Commission. Le Conseil du trésor aurait donc pu amorcer dès 1967 la mise en place des 82 districts bilingues. Les fonctionnaires n'ont pas proposé cette mesure parce qu'ils n'en appréciaient pas la valeur symbolique. Sans la Commission, l'appareil administratif aurait probablement maintenu son approche discrétionnaire en matière de "districtisation ». En fait, il a maintenu ses régions bilingues malgré les recommandations de la Commission. La déviation bureaucratique de 1976 et les règlements de 1992 en sont les meilleurs exemples.

Cela soulève une question essentielle : puisque la Commission se méfiait du Conseil du trésor et ne pensait pas que les régions bilingues discrètes pouvaient jouer le rôle symbolique des districts bilingues, pourquoi n'a-t-elle pas proposé de transformer les 82 régions bilingues en districts bilingues? La Commission a choisi de déléguer cette tâche à un conseil fédéral-provincial parce que ses membres préféraient les unités de recensement aux unités administratives en place, ne s'entendaient pas sur les districts bilingues 
délimités à partir des premiers et souhaitaient que les districts bilingues soient conjoints. La question se pose maintenant ainsi : puisque les fonctionnaires ont proposé des districts bilingues uniquement fédéraux, après avoir constaté que les provinces hésitaient à en faire un projet conjoint, comme la Commission l'avait prédit, pourquoi n'ont-ils pas proposé la transformation des 82 régions bilingues définies en 1966 en 82 districts bilingues en 1967 ?

La Commission a été dépassée par les événements et n’a pas su ou osé s'adapter au changement. Elle semblait figée dans sa méfiance à l'égard de l'appareil fédéral alors que celui-ci avançait à grands pas. Les progrès accomplis par cet appareil en 1966 et 1967 auraient dû atténuer cette méfiance et lui permettre de mieux expliquer l'importance des mesures symboliques et de se montrer plus pragmatique.

Les hauts fonctionnaires étaient bien plus favorables au bilinguisme que la Commission ne le pensait. Les mesures qu'ils ont proposées en 1966 rejoignaient celles que la Commission allait recommander plus tard. Ils étaient même prêts à aller plus loin que la Commission à certains égards. Ils ont joué un rôle positif lors de la rédaction de la Loi sur les langues officielles, surtout en ce qui concerne les districts bilingues. Enfin, leur réaction aux rapports des comités consultatifs était très positive.

Si l'appareil fédéral a accompli de grands progrès en 1966 et 1967 et suite aux autres propositions de la Commission, on a perdu de vue la valeur symbolique des districts bilingues, la pierre angulaire du système. Si la valeur symbolique des districts bilingues importe toujours, la Commission avait raison de les proposer en 1967 et le gouvernement fédéral a eu tort de les abandonner en 1976.

Malgré ces exceptions, les recommandations de la Commission se sont révélées justes dans l'ensemble. En effet, la plupart de ses recommandations ont été mises en ouvre et perdurent encore aujourd'hui. Le fait que l'appareil fédéral a produit des mesures semblables en 1966 et 1967 confirme la perspicacité des commissaires. 
La structuration de la mise en œuvre des mesures administratives a contribué à l'échec de certaines d'entre elles

Notre étude de cas des districts bilingues avait abordé le rôle des commissions d'enquête dans l'établissement et la spécification des politiques, les politiques symboliques et la déviation bureaucratique $^{116}$. A également fait l'objet d'étude la représentation de la diversité linguistique dans les administrations fédérales ${ }^{117}$. L'analyse qui suit porte sur la structuration de la mise en œuvre des mesures administratives et se fonde sur des théories des organisations.

Les organisations publiques sont établies pour atteindre des objectifs qui sont mieux réalisés de façon collective, mais elles sont si grosses qu'on doit fractionner leurs actions en unités ${ }^{118}$. Dans les États modernes, suivant l'autorité légale, le fractionnement de l'appareil administratif suit l'approche rationnelle ${ }^{119}$. On a érigé, par conséquent, des structures bureaucratiques selon une division du travail spécialisé ${ }^{120}$. Le développement de l'État providence au milieu $\mathrm{du} \mathrm{Xx}^{\mathrm{e}}$ siècle a engendré une multitude d'organisations publiques pour prendre en charge de nombreuses compétences, ce qui a eu

116 Bourgeois, La genèse, la spécification et l'abandon des districts bilingues canadiens, 1966-1976, p. 373-416.

117 Daniel Kübler, Émilienne Kobelt et Stéphanie Andrey, «Vers une bureaucratie représentative : la promotion de la représentation et de la diversité linguistiques dans l'administration fédérale en Suisse et au Canada ", Revue canadienne de science politique, vol. 44, $\mathrm{n}^{\circ} 4$ (décembre 2011), p. 903-927, [En ligne], [http://dx.doi.org/10.1017/S0008423911000801]; Luc Turgeon et Alain G. Gagnon, "The Politics of Representative Bureaucracy in Multilingual States: A Comparison of Belgium, Canada and Switzerland ", Regional and Federal Studies, vol. 23, n 4 (2013), p. 407-425, [En ligne], [http://dx.doi.org/10.1080/ 13597566.2013.765866].

118 Chris Argyris, Personality and Organization: The Conflict between System and the Individual, New York, Harper and Row, 1957.

119 Max Weber, The Theory of Social and Economic Organizations, New York, Free Press, 1947.

120 Frederick Winslow Taylor, The Principles of Scientific Management, New York, Harper and Brothers, 1911. 
pour effet de spécialiser davantage le travail entre ces organisations et de nourrir le besoin de créer des organismes de coordination ${ }^{121}$.

La modernisation de l'appareil fédéral entreprise par les gouvernements Pearson et Trudeau, suite au rapport de la commission Glassco, a maintenu le Bureau du Conseil privé en tant qu'organisme central, mais elle a élevé le Conseil du trésor à un statut quasiment similaire ${ }^{122}$. Le premier devait coordonner les politiques alors que le second devait assurer leur mise en œuvre ${ }^{123}$. Or ces chambardements rationnels de l'appareil fédéral mettront en péril l'existence des districts bilingues.

Le Secrétariat d'État était le ministère responsable des mesures prévues par la Loi sur les langues officielles, y compris les districts bilingues. Il offrait des programmes de promotion des langues officielles auprès des gouvernements provinciaux et des communautés minoritaires. Pour sa part, le Conseil du trésor était responsable de la mise en ouvre des mesures administratives, y compris les districts bilingues une fois définis. Les deux organismes avaient donc un mandat particulier et une culture organisationnelle différente. Cette différence n'aurait causé aucun ennui tant que la spécification et la mise en ouvre de la loi demeuraient des étapes autonomes.

Sachant que son ministère ne pouvait imposer de lignes directrices aux autres ministères en matière de prestation de services bilingues, suite à la proclamation des districts bilingues (et des régions bilingues), le secrétaire d'État demanda au Cabinet, en février 1972, de déléguer ce pouvoir au Conseil du trésor. Or, sachant aussi que le Conseil du trésor n'allait pas définir les districts bilingues, le secrétaire d'État en

121 J. E. Hodgetts, The Canadian Public Service: A Physiology of Government, $1867-$ 1970, Toronto, University of Toronto Press, 1973.

122 G. Bruce Doern, "Recent Changes in the Philosophy of Policy-making in Canada ", Canadian Journal of Political Science, vol. 4, no 2 (juin 1971), p. 243264, [En ligne], [http://dx.doi.org/10.1017/S0008423900026664].

123 Gordon Robertson, "The Changing Role of the Privy Council Office ", Canadian Public Administration, vol. 14, no 4 (décembre 1971), p. 506; A. W. Johnson, "The Treasury Board of Canada and the Machinery of Government of the 1970's ", Canadian Journal of Political Science, vol. 4, n 3 (septembre 1971), p. 346, [En ligne], [http://dx.doi.org/10.1017/S0008423900026810]. 
a conservé la responsabilité. Ainsi, l'organisme de protection des langues officielles demeurait responsable de la spécification des districts bilingues, menée par les conseils consultatifs, alors que le Conseil du trésor était responsable de les mettre sur pied et d'établir le système de postes bilingues nécessaires.

Toutefois, lorsque le rapport du premier Conseil consultatif a été mis de côté parce que les districts bilingues proposés en fonction du seuil de $20 \%$ étaient remis en question par les données du recensement de 1971, ce qui s'est pourtant révélé erroné par la suite, et lorsqu'un second Conseil consultatif a été mis sur pied, le Conseil du trésor a décidé de mettre à jour les régions bilingues de 1966 en fonction de l'importance de la demande en dehors des districts bilingues éventuels, en attendant leur proclamation. En fait, il a aussi décidé de prévoir les districts bilingues éventuels en ajoutant, à la liste de ses régions bilingues, certaines régions dont le statut était incontestable. Le Conseil du trésor voulait devancer le rapport du Conseil consultatif, mais en établissant ses régions bilingues selon l'article 9(2) et en y incluant des districts bilingues, il a établi un système bureaucratique trop onéreux pour le reléguer aux oubliettes. Il a abandonné les districts bilingues parce qu'il ne reconnaissait pas leur valeur symbolique. Le Secrétariat d'État, comprenant cette valeur symbolique, a conservé leur désignation. Toutefois, contre l'avis du Bureau du Conseil privé ${ }^{124}$, le Conseil du trésor a hérité du mémoire déposé au Cabinet et qui faisait suite au rapport du Conseil consultatif. Il en a profité pour éliminer le concept de district bilingue.

Pour être précis, les dirigeants du Conseil du trésor ont compris que les districts bilingues avaient une valeur symbolique, mais cette dernière était négligeable à leurs yeux ${ }^{125}$. D'abord, une seule province était prête à réaliser un projet conjoint. Ensuite, les minorités souhaitaient avoir accès à l'éducation dans leur langue davantage que des services

124 Clovis Demers, « Note à Monsieur Ross - Districts bilingues », Bureau du Conseil privé, Ottawa, 29 juin 1976.

125 Memorandum to Cabinet: Bilingual Districts and Related Measures, p. 36 
et des communications bilingues de la part du gouvernement fédéral. Enfin, les districts bilingues étaient des "ghettos " et des outils provocateurs (" red rag to a bull ", selon un dirigeant du Conseil du trésor), surtout au Québec. Le mémoire du second Conseil consultatif néglige de préciser que les minoritaires voulaient des districts bilingues et que le gouvernement du Québec acceptait leur proclamation.

Plusieurs fonctionnaires du Bureau du Conseil privé partageaient la crainte de la Commission et celle du Secrétariat d'État au sujet de la rigidité bureaucratique du Conseil du trésor. Ils se méfiaient même de la déviation orchestrée par ses dirigeants en novembre 1976. Nous n’avons pas pu déterminer les raisons pour lesquelles leurs mises en garde ne se sont pas rendues au Cabinet. Le Bureau du Conseil privé avait pourtant préséance sur le Conseil du trésor. On ne sait donc pas pourquoi les dirigeants du ministère responsable de la création des districts bilingues et ceux de l'agence centrale responsable de la coordination des politiques ne se sont pas opposés à la recommandation que leurs collègues du Conseil du trésor avaient faite au Cabinet en novembre 1976, laquelle visait à abandonner les districts bilingues.

Ces derniers auraient vu le jour si le Conseil du trésor ne s'était pas ingéré dans le processus. Le Secrétariat d'État avait la responsabilité du dossier jusqu'à la désignation et la proclamation initiale, mais le Conseil du trésor a outrepassé son mandat - les régions bilingues définies selon l'importance de la demande, prévue à l'article 9(2) - et a opéré une déviation bureaucratique dans le but d'empêcher la proclamation de districts bilingues et se décharger du lourd fardeau administratif nécessaire à leur mise en œuvre. Les hauts fonctionnaires du Conseil du trésor ont donc détruit la pierre angulaire pour éviter de consacrer plus de temps à refaire son système de prestation des services et des communications bilingues au sein des régions bilingues. Au lieu d'attendre le rapport du Conseil consultatif et la proclamation des premiers districts bilingues par le Cabinet, il a décidé d'inclure dans les régions bilingues ce qu'il prévoyait être les districts bilingues. Or, une fois le système achevé, ses dirigeants ont constaté que la mise en ouvre des districts bilingues pouvait le chambarder. 
La structuration du suivi aux mesures proposées par la Commission a donc contribué à l'échec des districts bilingues. Les théories des organisations ont bien montré l'importance des tensions intraorganisationnelles entre l'autorité formelle fondée sur l'expertise et la hiérarchie et l'autorité informelle, fondée sur la discrétion et les jeux de pouvoir ${ }^{126}$. Notre étude de cas montre que deux expertises étaient en jeu et que l'expertise administrative du Conseil du trésor a eu le dessus sur l'expertise du Secrétariat d'État en matière symbolique, en partie grâce à des jeux de pouvoir. On ignore comment le Conseil du trésor a pu ravir au Secrétariat d'État, contre l'avis du Bureau du Conseil privé, le mandat d'assurer le suivi au rapport du Conseil consultatif.

Les théories des organisations ont aussi montré l'existence de tensions entre les organismes d'une même entité ${ }^{127}$. Ces tensions interorganisationnelles se manifestent entre ministères fonctionnels (line departments) et agences centrales. Ces dernières sont des organes de gestion qui assument la responsabilité collective du Cabinet : "[They] play a watchdog role over the bureaucracy on behalf of the government for the efficiency and effectiveness of bureaucratic process as opposed to the substance of policy ${ }^{128}$. " Dans le cas des districts bilingues, toutefois, le Conseil du trésor n'a pas joué son rôle d'agence centrale; il a agi comme ministère responsable d'un dossier qui a défendu ses intérêts organisationnels. Sa complexité administrative et son apogée comme agence centrale à l'aboutissement de la rationalisation de l'appareil fédéral ${ }^{129}$ lui donnaient un avantage sur les ministères

126 Alvin Ward Gouldner, Patterns of Industrial Bureaucracy, Glenco, Free Press, 1954; Michel Crozier et Erhard Friedberg, L'acteur et le système : les contraintes de l'action collective, Paris, Éditions du Seuil, 1977.

127 Robert K. Merton, "Social Structure and Anomie ", American Sociological Review, vol. 3, no 5 (octobre 1938), p. 672-682.

128 Sharon Lynn Sutherland et G. Bruce Doern, Bureaucracy in Canada: Control and Reform, Toronto, University of Toronto Press, 1985.

129 Gérard Veilleux et Donald J. Savoie, "Kafka's Castle: The Treasury Board of Canada Revisited ", Canadian Public Administration, vol. 31, n 4 (décembre 1988), p. 527. 
sectoriels tel le Secrétariat d'État. Pourtant, le Conseil du trésor n'avait aucune expertise dans le domaine des politiques symboliques. Le Bureau du Conseil privé n'a pas été un bon chien de garde. Il aurait dû rappeler au Cabinet que la commission BB avait proposé des districts bilingues parce que les régions bilingues ne suffisaient pas. Fait inusité : une agence centrale devait donc en surveiller une autre. Le Conseil du trésor a donc saboté le concept parce que le permettait la structuration des responsabilités. Une structure efficace est donc essentielle à la mise en œuvre des politiques.

Il faut cependant nuancer notre conclusion. La structuration officielle était adéquate : le Secrétariat d'État conservait la responsabilité des districts bilingues jusqu'à leur proclamation, avant de les confier au Conseil du trésor, organisme responsable des régions bilingues à l'extérieur de ces districts. Le Conseil du trésor devait donc définir les régions en attendant la proclamation des districts. Lorsque le Secrétariat d'État demanda au Cabinet de transférer au Conseil du trésor la mise en ouvre des districts bilingues, il ne s'attendait pas à ce que le Conseil du trésor élargisse son mandat pour établir des régions bilingues où il prévoyait l'établissement éventuel de districts bilingues. Lorsque le Conseil du trésor a ainsi élargi son mandat, il a mis en place un système de prestation de services bilingues et de désignation de postes bilingues trop complexe et onéreux pour l'abandonner à la proclamation de districts bilingues. On ne connaît pas les raisons pour lesquelles le Secrétariat d'État et le Bureau du Conseil privé sont restés muets lorsque le Conseil du trésor s'est opposé avec succès à la valeur symbolique du concept. La structure des responsabilités était donc adéquate en partant, mais elle a fait l'objet d'une déviation bureaucratique. La modification de février 1972 à la structure décisionnelle initiale a contribué à l'abandon des districts bilingues. 
Il ne faut toutefois pas négliger l'imputabilité des sous-ministres dans les déviations ${ }^{130}$. Le cas des districts bilingues indique que le sous-ministre du Secrétariat d'État et le greffier du Conseil privé n'ont pas cherché à rendre imputable le secrétaire du Conseil du trésor. Nous en ignorons la raison.

\section{Conclusion}

La Commission a bien étudié l'appareil fédéral et a proposé des mesures pour lui permettre de relever plusieurs défis importants en matière de langues officielles. La plupart de ses mesures ont été mises en œuvre et perdurent de nos jours. Le fait que l'appareil fédéral a proposé des mesures semblables au même moment confirme l'excellente compréhension de la Commission concernant cet appareil.

Néanmoins, la Commission se méfiait de ce bastion anglophone incapable d'innover pour relever les défis auxquels faisait face le Canada. Cette méfiance a été à la fois infirmée et confirmée par la suite. D'une part, alors que la Commission faisait son travail, la bureaucratie fédérale a proposé plusieurs mesures qui rejoignaient celles que la Commission allait proposer plus tard. En fait, elles ont quasiment rendues désuètes plusieurs recommandations de la Commission. D'autre part, l'appareil gouvernemental n'a pas compris la valeur symbolique des districts bilingues et les a écartés. La Commission aurait toutefois pu proposer la proclamation officielle des 82 régions bilingues. Elle aurait ainsi évité la structuration problématique de la mise en œuvre des districts bilingues.

130 Gordon F. Osbaldeston, Keeping Deputy Ministers Accountable, Toronto, McGrawHill Ryerson, 1989. Ironie du sort, Osbaldeston a été le principal responsable de l'élimination des districts bilingues. 